\title{
OPTICAL
} PROPERTIES

\section{Luminescence and Electronic Excitations in Crystals $\mathbf{K}_{2} \mathrm{Al}_{\mathbf{2}} \mathbf{B}_{\mathbf{2}} \mathrm{O}_{7}$ with Defects}

\author{
I. N. Ogorodnikov ${ }^{a}$ *, V. A. Pustovarov ${ }^{a}$, S. A. Yakovlev ${ }^{a}$, \\ L. I. Isaenko ${ }^{b}$, and S. A. Zhurkov ${ }^{b}$ \\ ${ }^{a}$ Yeltsin Ural Federal University, ul. Mira 19, Yekaterinburg, 620002 Russia \\ *e-mail:ogo@dpt.ustu.ru \\ ${ }^{b}$ Sobolev Institute of Geology and Mineralogy, Siberian Branch, Russian Academy of Science, \\ pr. Akad. Koptyuga 3, Novosibirsk, 630090 Russia
}

Received May 17, 2011

\begin{abstract}
This paper reports on the results of the comprehensive study of the dynamics of electronic excitations in $\mathrm{K}_{2} \mathrm{Al}_{2} \mathrm{~B}_{2} \mathrm{O}_{7}$ (KABO) crystals, obtained by low-temperature luminescence vacuum ultraviolet spectroscopy with nanosecond time resolution upon photoexcitation by synchrotron radiation. For the first time, the data have been obtained on the photoluminescence (PL) decay kinetics, PL spectra with time resolution, PL excitation spectra with time resolution, and reflection spectra at $7 \mathrm{~K}$; the intrinsic nature of PL at $3.28 \mathrm{eV}$ has been established; luminescence bands of defects have been separated in the visible and ultraviolet spectral regions; an intense long-wavelength PL band has been detected at $1.72 \mathrm{eV}$; channels of the formation and decay of electronic excitations in $\mathrm{K}_{2} \mathrm{Al}_{2} \mathrm{~B}_{2} \mathrm{O}_{7}$ crystals have been discussed.
\end{abstract}

DOI: $10.1134 / \mathrm{S} 1063783412010258$

\section{INTRODUCTION}

Optical low-symmetry crystals have been widely used in modern short-wavelength laser engineering and integrated optics as nonlinear transducers, parametric oscillators, and other optical elements. In this respect, $\mathrm{K}_{2} \mathrm{Al}_{2} \mathrm{~B}_{2} \mathrm{O}_{7}(\mathrm{KABO})$ crystals represent a relatively new nonlinear-optical material developed for applications in a wide spectral range from 180 to $3600 \mathrm{~nm}$. They are nonhygroscopic, have a density of $2.46 \mathrm{~g} \mathrm{~cm}^{-3}$, and feature mechanical strength [1-3]. KAVO crystals have been used to develop high-power sources of coherent radiation of ultraviolet (UV) and vacuum ultraviolet (VUV) spectral ranges by generation of the fourth harmonic $(266 \mathrm{~nm})$ and sum frequencies (to $193 \mathrm{~nm}$ ) of radiations from $\mathrm{Nd}^{3+}$ ion lasers [4-8].

From the crystallographic point of view, KABO crystals belong to the trigonal crystal system (space group $P 32_{1}$ ), the unit cell contains three formula units ( 39 atoms) and is characterized by the parameters $a=$ $b=0.85669(8) \mathrm{nm}, c=0.8467(1) \mathrm{nm}$, and $\gamma=120^{\circ}$. A layered structure is characteristic of $\mathrm{KABO}$ : the main structural motif is a layer of composition $\mathrm{Al}_{2}\left(\mathrm{BO}_{3}\right)_{2} \mathrm{O}$ formed by distorted $\mathrm{AlO}_{4}$ tetrahedra and $\mathrm{BO}_{3}$ triangles whose planes are oriented almost parallel to the $c$ axis [9]. Bridge oxygen ions join neighboring layers into a 3D aluminum-boron-oxygen framework whose voids contain potassium cations playing the role of structure stabilizers [10-13].
The serious problem complicating practical application of KABO is optical absorption of the crystal in the range of 190-280 $\mathrm{nm}$, caused by impurity iron ions [1]. Impurity defects based on $\mathrm{Fe}^{3+}$ ions in KABO crystals were identified by electron paramagnetic resonance and optical absorption methods [14, 15]. However, we know no data on luminescence spectroscopy of KABO crystals and defects in them.

The objective of this research is to study the formation and evolution of electronic excitations in KABO crystals using low-temperature vacuum ultraviolet time-resolved luminescence spectroscopy methods upon selective excitation by synchrotron radiation.

\section{EXPERIMENTAL DETAILS}

We studied KABO crystals of optical quality, grown at the Institute of Geology and Mineralogy (Novosibirsk) by the solution-melt method with an upper seed position. As initial reagents, ultrapure-grade $\mathrm{K}_{2} \mathrm{CO}_{3}, \mathrm{Al}_{2} \mathrm{O}_{3}$, and $\mathrm{H}_{3} \mathrm{BO}_{3}$ were used. A KABO powder was obtained by solid-phase synthesis from preliminarily annealed (at $400^{\circ} \mathrm{C}$ ) powders of initial reagents taken in the stoichiometric ratio,

$$
\begin{aligned}
& \mathrm{K}_{2} \mathrm{CO}_{3}+\mathrm{Al}_{2} \mathrm{O}_{3}+2 \mathrm{H}_{3} \mathrm{BO}_{3} \\
= & \mathrm{K}_{2} \mathrm{Al}_{2} \mathrm{~B}_{2} \mathrm{O}_{7}+\mathrm{CO}_{2}+2 \mathrm{H}_{2} \mathrm{O} .
\end{aligned}
$$

To grow the $\mathrm{K}_{2} \mathrm{Al}_{2} \mathrm{~B}_{2} \mathrm{O}_{7}$ crystal, a preliminarily synthesized $\mathrm{KABO}$ powder mixed with a $\mathrm{KBF}_{4}$ solvent in the molar ratio of $4: 1$ was loaded into a platinum cru- 


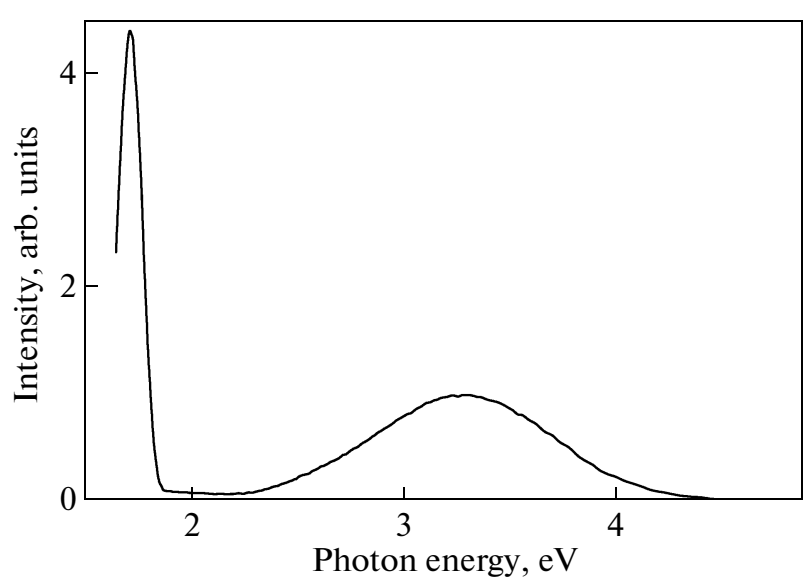

Fig. 1. KABO crystal PL survey spectrum in the range of $1.5-5.0 \mathrm{eV}$ of at $T=7 \mathrm{~K}$ and $E_{\mathrm{ex}}=6.78 \mathrm{eV}$.

cible $40 \mathrm{~mm}$ in diameter and $50 \mathrm{~mm}$ high. The total mixture weight was $50 \mathrm{~g}$. The crucible was placed into a growth furnace and heated for $12 \mathrm{~h}$ to a temperature of $950^{\circ} \mathrm{C}$; in this case, the composition was completely molten. The melt was kept at this temperature for $4 \mathrm{~h}$ for complete homogenization. The KABO crystal was grown on a seed oriented in the [110] direction at an initial temperature of $920^{\circ} \mathrm{C}$; then the temperature was decreased with a rate of $2-4^{\circ} \mathrm{C}$ per day, which provided growth of a hexagonal transparent plate $20 \mathrm{~mm}$ in size and $1.5 \mathrm{~mm}$ thick. Samples for spectroscopic studies were prepared as plane-parallel transparent plates $6 \times 5 \times 1 \mathrm{~mm}$ in size.

The time-resolved photoluminescence (PL) spectra in the spectral range of 1.2-6.2 eV, time-resolved PL excitation spectra $(3.7-21 \mathrm{eV})$, spectra of reflection at an angle of $17^{\circ}$, and PL decay kinetics with subnanosecond time resolution were measured upon selective photoexcitation by synchrotron radiation (SR) at the SUPERLUMI experimental station [16] of the HASYLAB laboratory. SR pulses of the DORIS storage ring was Gaussian-shaped (FWHM $=130 \mathrm{ps})$ with a repetition period of 96 ns. Measurements were performed at a temperature of $7 \mathrm{~K}$ using a continuous flow helium cryostat providing a vacuum no worse than $7 \times 10^{-10}$ Torr. The time-resolved spectra were measured in a time window of width $\Delta t$ delayed relative to the exciting SR pulse by $\delta t$. In this study, simultaneous measurements were performed in two independent windows with parameters $\delta t_{1}=0.5 \mathrm{~ns}, \Delta t_{1}=$ $2.3 \mathrm{~ns}$ (fast component) and $\delta t_{2}=14 \mathrm{~ns}, \Delta t_{2}=58 \mathrm{~ns}$ (slow component). Simultaneously, the PL spectrum without time resolution (time-integrated spectrum) was measured. Selective excitation of PL by synchrotron radiation in the range of $3.7-21 \mathrm{eV}$ was performed using a $2 m$-vacuum monochromator with an $\mathrm{Al}$ grating (the spectral resolution is $0.32 \mathrm{~nm}$ ). The $\mathrm{PL}$ excitation spectra were normalized to the equal num-

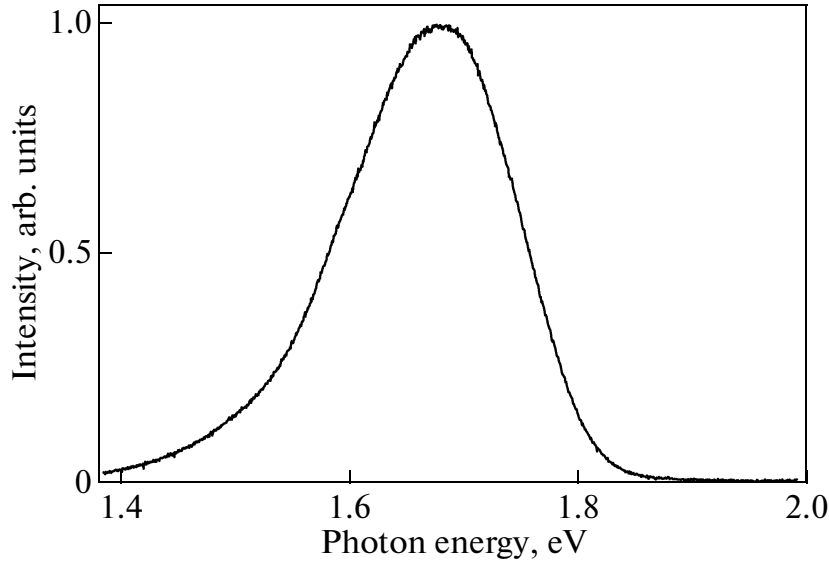

Fig. 2. KABO crystal PL spectrum in the range of 1.4$2.0 \mathrm{eV}$ at $T=7 \mathrm{~K}$ and $E_{\mathrm{ex}}=6.78 \mathrm{eV}$, measured using a CCD camera.

ber of photons incident on the sample. The PL spectra in the range of 1.5-6.2 eV were measured using a ARC Spectra Pro-308i 0.3m-monochromator, an R6358P (Hamamatsu) photomultiplier (PM) or a CCD camera. The PL spectra were not corrected for the spectral sensitivity of the optical channel. Fast components of the PL decay kinetics were analyzed using the convolution integral method when considering the instrumental profile of the excitation pulse (the full width at half-maximum is $\sim 300 \mathrm{ps}$ ).

\section{EXPERIMENTAL RESULTS}

Low-temperature luminescence of KABO crystals was studied in a wide spectral range from 1.4 to $6.2 \mathrm{eV}$ upon selective excitation by photons of various energies $E_{\text {ex }}$. Figure 1 shows the survey PL spectrum in the range of $1.5-5.0 \mathrm{eV}$ of the $\mathrm{KABO}$ crystal at $7 \mathrm{~K}$. In the low-temperature spectral region, a strong PL band at $1.72 \mathrm{eV}$ was detected. This band is near the low-energy boundary of the studied spectral range, which did not allow detailed study of its shape. Figure 2 shows the spectrum of the long-wavelength PL band, measured using a CCD camera. The luminescence band maximum is observed at $1.677 \mathrm{eV}$, which is caused by differences in the spectral sensitivity of the PM and CCD camera in this spectral region. Another group of intense PL bands is localized in the range of 2.0$4.5 \mathrm{eV}$ (Fig. 3). In the high-energy spectral range of 4.5-6.2 eV, luminescence at $7 \mathrm{~K}$ is not observed. Therefore, we enlarge on the discussion of PL spectra in the visible and UV spectral regions.

Figure 3 shows the KABO PL spectra measured at $T=7 \mathrm{~K}$. Upon excitation in the crystal transmission band $\left(E_{\mathrm{ex}}=4.28 \mathrm{eV}\right), \mathrm{PL}$ is observed, whose spectrum is presented by a low-intensity broad band in the range of $2.0-4.5 \mathrm{eV}$, consisting of two elementary Gaussian bands at 2.68 and $3.54 \mathrm{eV}$ (see table). The fact of PL excitation in the crystal transmission band makes it 


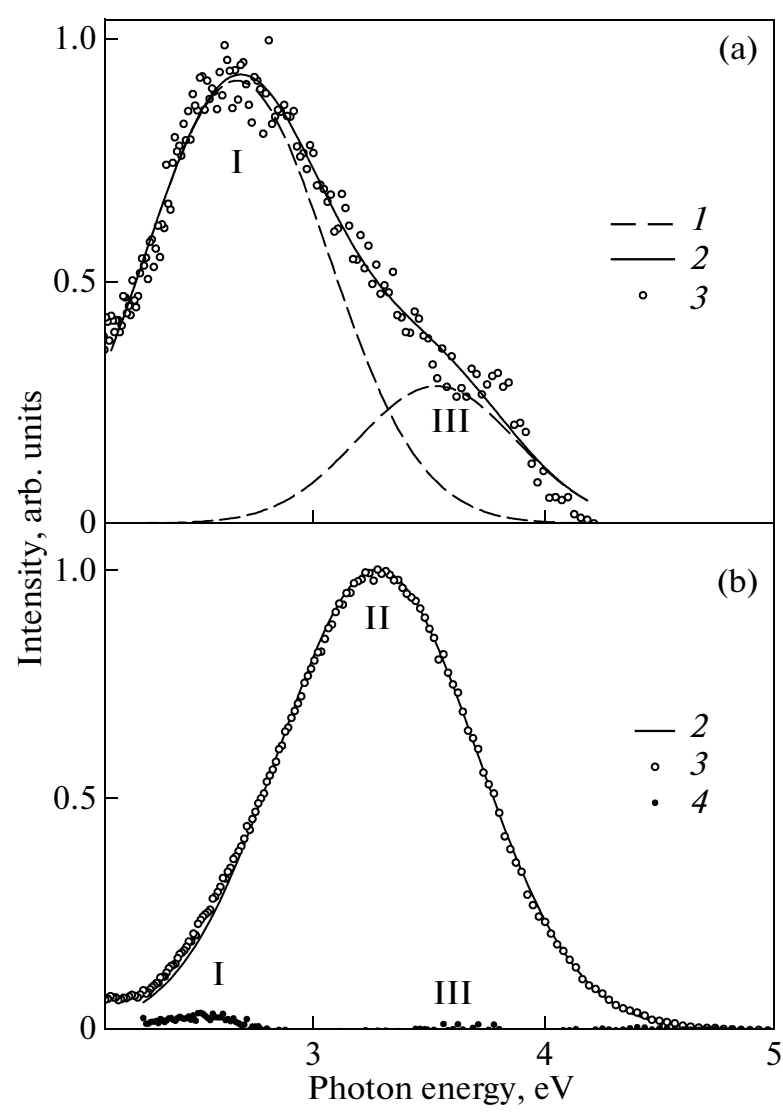

Fig. 3. KABO crystal PL spectrum in the visible and UV regions at $T=7 \mathrm{~K}$ and $E_{\mathrm{ex}}=$ (a) $4.28 \mathrm{eV}$ and (b) $6.78 \mathrm{eV}$. (1) Main elementary Gaussian-shaped bands, (2) result of approximation, (3) experimental data, and (4) deviation of experimental data from approximation curve.

possible to attribute this luminescence to optical transitions in intrinsic or impurity defects of the KABO lattice. Upon excitation in the crystal fundamental absorption band $\left(E_{\mathrm{ex}}=6.78 \mathrm{eV}\right)$, the intense broad PL band dominates at $3.28 \mathrm{eV}$ in the spectrum, which is approximated a single Gaussian band. Insignificant (about one percent) deviations of experimental data from the approximating curve (Fig. 3) are observed exactly in the spectral regions corresponding to defect luminescence bands at 2.68 and $3.54 \mathrm{eV}$. This indicates the extremely low but nonzero PL excitation efficiency for KABO defects in the fundamental absorption band. In all cases considered, we measured timeresolved PL spectra in both time windows and timeintegrated PL spectra. The profiles of all spectra were identical; therefore, only time-integrated PL spectra are shown in Figs. 1 and 3.

Figure 4 shows the PL decay kinetics measured upon selective excitation by photons of various energies. Upon excitation by photons with energies of $6.78-6.88 \mathrm{eV}$, the kinetics with a time constant of micro- and millisecond ranges is observed, which is shown as a pedestal. Upon excitation in the crystal

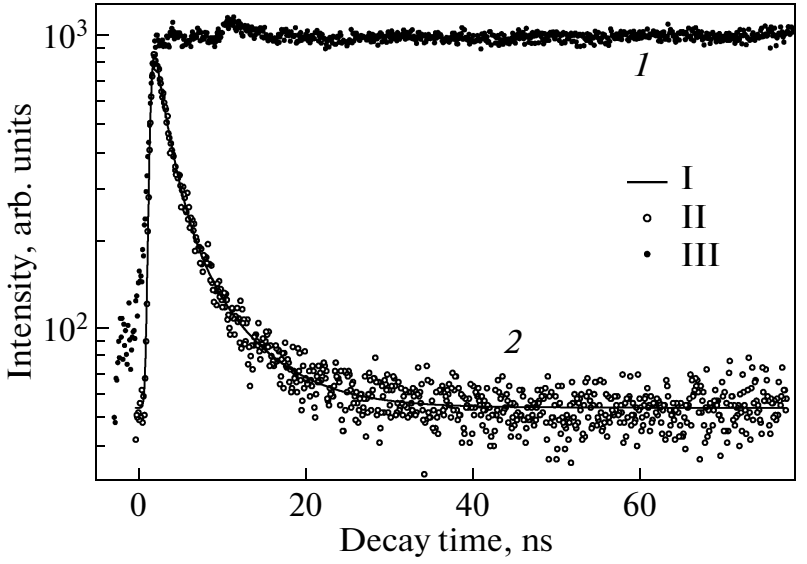

Fig. 4. PL decay kinetics of the KABO crystal at $7 \mathrm{~K}$, measured in the luminescence band at $2.90 \mathrm{eV}$ for excitation photon energies of (1) 6.8 and (2) $4.1 \mathrm{eV}$. Solid curve I is the approximation result; open and closed circles II and III are experimental data.

optical transparency band, the PL decay kinetics consists of two exponential components with time constants of 1.8 and $6.5 \mathrm{~ns}$ and a pedestal. The ratio of intensities of these components is $100: 30: 13$, respectively.

Figures 5 and 6 show the PL excitation spectra in four various luminescence bands (the long-wavelength band at $1.72 \mathrm{eV}$ and three bands of visible and UV regions, denoted by numerals I, II, and III in Fig. 3, respectively), and the reflection spectrum of the $\mathrm{KABO}$ crystal at $7 \mathrm{~K}$. At $E_{\mathrm{ex}}>6.5 \mathrm{eV}$, the profiles of excitation spectra of all PL bands are identical: at $6.8 \mathrm{eV}$, the strong narrow maximum (main peak) is observed. Then a sharp slope at $7 \mathrm{eV}$ and a broad lowintensity maximum in the range of $7-10 \mathrm{eV}$ are observed. In the range of $10-18 \mathrm{eV}$, the excitation efficiency is extremely low; as the excitation photon energy further increases, the PL excitation efficiency monotonically increases.

Despite the general similarity, all excitation spectra differ in details. The most significant differences are as follows. The low-energy slope of the main peak of the

Parameters of PL bands of the KABO crystal at $7 \mathrm{~K}$

\begin{tabular}{l|c|c|c}
\hline \multirow{2}{*}{ Parameter } & \multicolumn{3}{|c}{ PL band } \\
\cline { 2 - 4 } & I & II & III \\
\hline$E_{\mathrm{ex}}, \mathrm{eV}$ & 4.28 & 6.78 & 4.28 \\
$E_{m}, \mathrm{eV}$ & 2.68 & 3.28 & 3.54 \\
$\Delta E, \mathrm{eV}$ & 0.93 & 1.0 & 0.80 \\
$S, \%$ & 90.5 & 99.0 & 24.3 \\
\hline
\end{tabular}

Note: $\Delta E$ is the PL band width at half maximum (FWHM); $S$ is the contribution of the PL band to the total light yield of the observed PL. 


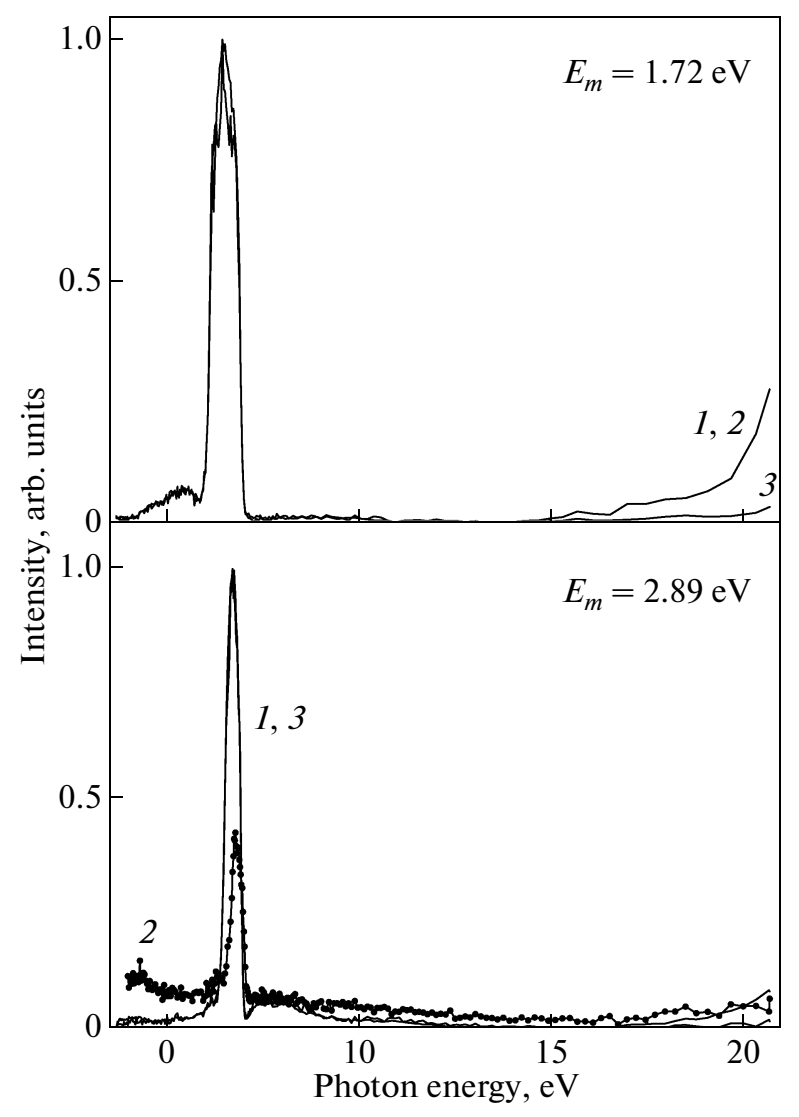

Fig. 5. Excitation spectra of (1) time-integrated, (2) fast, and (3) slow PL components in luminescence bands at $E_{m}=1.72$ and $2.89 \mathrm{eV}$ of the KABO crystal at $7 \mathrm{~K}$.

excitation spectrum of the long-wavelength PL band is broadened to the crystal optical transparency region. The wide low-intensity maximum in the range of 7$10 \mathrm{eV}$ is strongest and most structured for the excitation spectrum of band II. The excitation spectra of the fast and time-integrated PL components have identical profiles in all cases, except for the excitation spectrum of band I. In the high-energy region, the excitation spectrum of the slow component differs appreciably from the excitation spectra of the fast and timeintegrated PL components. In this case, the most significant differences are observed for the excitation spectra of band II. In the crystal transparency region, only luminescence bands I, III, and long-wavelength band are excited. In this case, the fast component dominates in time-resolved spectra.

\section{DISCUSSION}

The data of quantum chemical calculations the $\mathrm{KABO}$ electronic structure $[15,17]$ suggest that the upper states of the KABO valence band are formed mostly by $2 p$-orbitals of oxygen ions, and the bottom of the conduction band is formed by $4 s$-orbitals of potassium ions. The lowest-energy electronic excita-

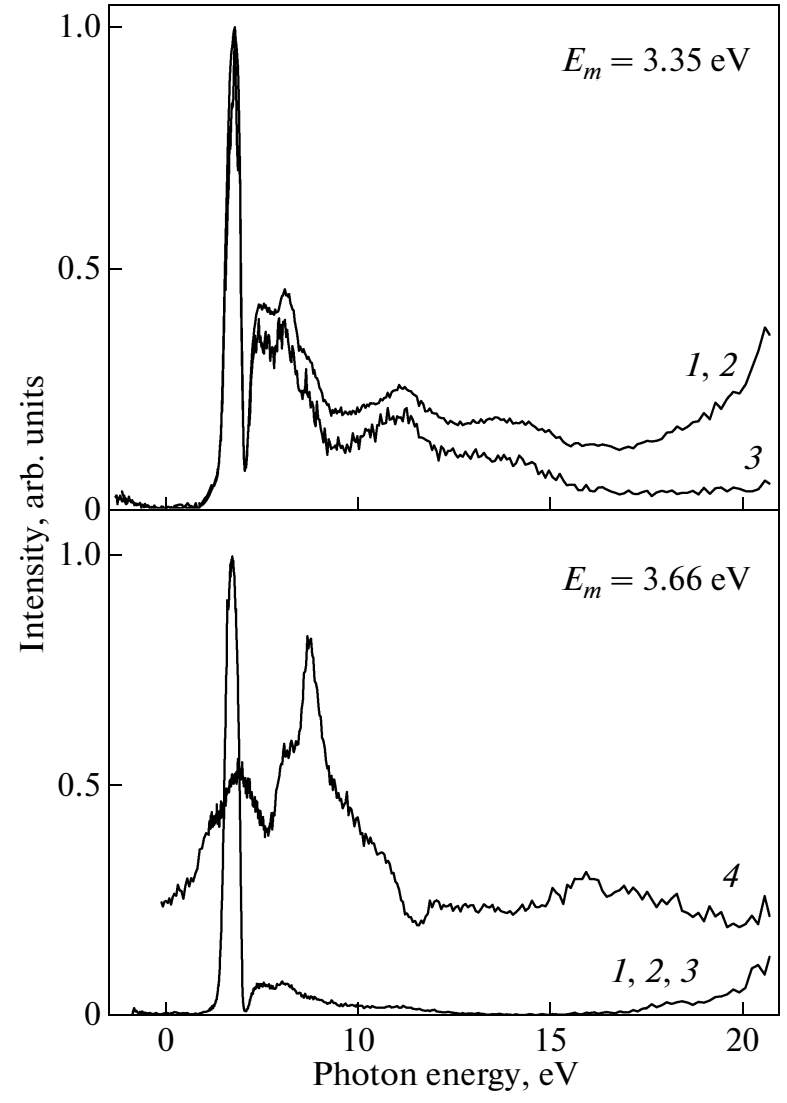

Fig. 6. (1-3) Excitation spectra of (1) time-integrated, (2) fast, and (3) slow PL components in luminescence bands at $E_{m}=3.35$ and $3.66 \mathrm{eV}$ and (4) the reflection spectrum of the $\mathrm{KABO}$ crystal at $7 \mathrm{~K}$.

tions in KABO crystals are caused by electron transfer from states of the top of the valence band to states of the bottom of the conduction band, i.e., by the electronic transition $\mathrm{O} 2 p \longrightarrow \mathrm{K} 4 s$.

The monotonic exponential growth of the optical absorbance of undoped KABO crystals, comparable to the long-wavelength fundamental absorption edge, is observed at photon energies above $6.6 \mathrm{eV}$ [14]. In the $\mathrm{KABO}$ reflection spectrum (Fig. 6), the energy position of the lowest-energy peak at $6.9 \mathrm{eV}$ is in the region of the comparatively narrow intense band with a maximum at $6.75 \mathrm{eV}$ in the PL excitation spectrum and is comparable to the formation of unrelaxed electronic excitations in the crystal. Indeed, exciton absorption in complex low-symmetry oxides is very often presented only by a shoulder or a "hump" at the longwavelength fundamental absorption edge; nevertheless, exciton features of the reflection spectrum are comparable to the region of the most efficient excitation of exciton luminescence [18]. A comparison of KABO reflection spectra (Fig. 6) with experimental and theoretical data on other borates (e.g., $\mathrm{Li}_{2} \mathrm{BO}_{4}$ $[18,19])$ allows a meaningful estimate of the KABO band gap as $E_{g}=8.0-8.5 \mathrm{eV}$. It can be assumed that 
excitation by photons in the KABO fundamental absorption edge results in the production of unrelaxed excitons $e^{\circ}$ whose most probable decay channels are migration followed by nonradiative decay, self-trapping followed by radiative annihilation, and dissociation into an electron and a hole with probable involvement of the latter in the recombination process. Let us discuss these $e^{\circ}$ decay channels in view of experimental data.

Migration and nonradiative decay on the surface cause the so-called surface energy loss. The profile of the KABO PL excitation spectrum is typical of borate crystals and points to the exciton energy transfer mechanism. This is characteristic of the systems with exciton luminescence mechanism, or excitons are intermediate during the energy transfer to the luminescence center $[19,20]$. Indeed, KABO luminescence at a temperature of $8 \mathrm{~K}$ is excited mostly in the exciton region: the main PL excitation band being a dominant comparatively narrow peak at $6.85-6.90 \mathrm{eV}$ (Fig. 6) is at the long-wavelength fundamental absorption edge of the KABO crystal in the presupposed region of free exciton formation. The profile of the low-energy slope of the PL excitation band is comparable to the long-wavelength edge of fundamental optical absorption; as the photon energy increases, it corresponds to an increase in the free exciton excitation efficiency due to an increase in the crystal optical absorbance. The high-energy decrease in the PL excitation band corresponds to a decrease in the photon mean free path in the crystal, hence, to an increase in the fraction of nonradiative energy losses of electronic excitations near the crystal surface. A comparison to the reflection spectrum profile suggests that the PL excitation spectrum structurization at $E_{\mathrm{ex}}>7 \mathrm{eV}$ is caused by modulation of PL excitation spectra by optical absorption of the crystal. The decrease in the luminescence excitation efficiency at photon energies above $10-12 \mathrm{eV}$ points to high mobility of electronic excitations and the presence of surface energy losses due to the significant increase in the optical absorbance. The monotonic increase in the excitation efficiency of low-temperature luminescence at energies above $18 \mathrm{eV}$ (Figs. 5 and 6) can be compared to the photon multiplication process, as was detected previously for some other wide band-gap oxides, e.g., for $\mathrm{BBO}$ [21], $\mathrm{Al}_{2} \mathrm{O}_{3}$ [22], or LBO [18, 20, 23], KDP [24]. However, this aspect requires detailed consideration.

Currently, exciton self-trapping (EST) followed by radiative decay are detected for many borates of alkali and alkaline-earth metals [18-20, 25, 26]. Band II in the KABO spectrum (Fig. 3b) exhibit all features of luminescence of relaxed electronic excitations, characteristic of other borates, i.e., the high enough quantum yield, the absence of selective excitation bands in the transparency region, efficient excitation in the region of presupposed exciton absorption, Stokes shift $(3.5 \mathrm{eV})$, and large half-width of the PL band, which indicate vibrational relaxation at the luminescence center. As is known, such a set of properties is inherent to EST in alkali halide crystals [27] or in wide bandgap low-symmetry oxides, e.g., in $\mathrm{BeO}[28,29]$ and $\mathrm{Al}_{2} \mathrm{O}_{3}$ [30]. This give reason to believe that the broad luminescence band at $3.28 \mathrm{eV}$ in KABO crystals is caused by radiative annihilation of relaxed electronic excitations, i.e., self-trapped excitons. The high efficiency of excitation of this luminescence immediately at the fundamental absorption edge of the crystal suggests that EST results from exciton $e^{\circ}$ relaxation. The absence of EST luminescence "freezing" at low temperatures $(T=7 \mathrm{~K})$ implies the absence of self-trapping barrier or its smallness, which is typical of many low-symmetry oxides with complex lattice, e.g., for $\mathrm{Al}_{2} \mathrm{O}_{3}[30]$.

The set of properties suggest that bands I and III of KABO PL should be attributed to luminescence of defects. An analysis of the available data on KABO crystal defects $[14,15]$ based on $\mathrm{Fe}^{3+}$ impurity ions can be considered as the most probable models of luminescence centers causing photoluminescence bands I and II. According to quantum-chemical calculations [14], $\mathrm{Fe}^{3+}$ impurity introduction to the regular $\mathrm{Al}^{3+}$ ion site results in the appearance of impurity states formed by Fe $3 d$-orbitals in the band gap. Optical transitions between states of the top of the valence band and impurity orbitals, $\mathrm{O} 2 p \rightarrow \mathrm{Fe} 2 d$ cause optical absorption bands at 4.7, 5.7, and $6.5 \mathrm{eV}$, corresponding to $\mathrm{Fe}^{3+}$ ion absorption in various KABO lattice environments [15]. Exactly in this energy range, bands I and III of defect luminescence in KABO are excited (Fig. 5).

The fast exponential kinetics of KABO PL decay is observed upon intracenter photoexcitation of defect luminescence (Fig. 4). The decay time constants of this kinetics are comparable to the radiative lifetime of the luminescence center in the excited state. Upon excitation into the fundamental absorption band, the slow PL decay kinetics is caused by the features of the electronic excitation energy transfer to KABO impurity centers.

The strong PL band at $1.72 \mathrm{eV}$ is most efficiently excited in the exciton region. This means that there is efficient transport of electronic excitation energy to luminescence centers of long-wavelength PL. The fact of broadening the main peak of the excitation spectrum to the crystal transparency region can indicate the formation of near-defect (near-impurity) excitons. The presence of selective excitation bands in the transparency region also indicates the possible relation of luminescence centers of long-wavelength PL to intrinsic or impurity defects of the KABO crystal lattice. The nature of the KABO long-wavelength PL band requires further study. At this stage, we only note the similarity of the KABO long-wavelength PL band to the PL band at $1.79 \mathrm{eV}$ in feldspar single crystals, 
caused by radiative transitions ${ }^{4} T_{1} \longrightarrow{ }^{6} A_{1}$ in $\mathrm{Fe}^{3+}$ ions arranged at $\mathrm{Al}^{3+}$ sites of the feldspar structure [31].

\section{CONCLUSIONS}

Thus, KABO crystals were studied for the first time using low-temperature luminescence-optical vacuum ultraviolet spectroscopy with nanosecond time resolution. Based on the set of experimental data obtained for the first time on the photoluminescence decay kinetics, PL spectra with time resolution, PL excitation spectra with time resolution, and reflection spectra at $7 \mathrm{~K}$, the intrinsic nature of PL at $3.28 \mathrm{eV}$ (band II) was established, defect luminescence bands at $2.68 \mathrm{eV}$ (band I) and at $3.54 \mathrm{eV}$ (band III) were separated. The channels of the formation and decay of electronic excitations in KABO crystals and the luminescence center nature were discussed. According to the developed concepts, PL band II is caused by intrinsic luminescence of the $\mathrm{KABO}$ crystal and is presumably attributed to radiative annihilation of selftrapped excitons. The strong long-wavelength PL band at $1.72 \mathrm{eV}$ was detected. Defect luminescence bands in the visible and ultraviolet spectral region are presumably attributed to radiative transitions at impurity centers based on $\mathrm{Fe}^{3+}$ ions in various environments of the KABO lattice.

\section{ACKNOWLEDGMENTS}

This study was supported in part by the HASYLAB DESY (project no. 20080019).

\section{REFERENCES}

1. N. Ye, W. Zeng, B. Wu, and C. Chen, Proc. SPIE-Int. Soc. Opt. Eng. 3556, 21 (1998).

2. Z. G. Hu, Y. Mori, T. Higashiyama, M. Yoshimura, Y. K. Yap, Y. Kagebayash, and T. Sasaki, Proc. SPIEInt. Soc. Opt. Eng. 3556, 156 (1998).

3. J. A. Kaduk, L. C. Satek, and S. T. Mckenna, Rigaku J. 16, 17 (1999).

4. N. Ye, W. Zeng, J. Jiang, B. Wu, C. Chen, B. Feng, and X. Zhang, J. Opt. Soc. Am. B 17, 764 (2000).

5. N. Umemura, M. Ando, K. Suzuki, E. Takaoka, K. Kato, Z.-G. Hu, M. Yoshimura, Y. Mori, and T. Sasaki, Appl. Opt. 42, 2716 (2003).

6. J. Lu, G. Wang, Z. Xu, C. Chen, J. Wang, C. Zhang, and Y. Liu, Chin. Phys. Lett. 19, 680 (2002).

7. P. Kumbhakar, S. Adachi, Z.-G. Hu, M. Yoshimura, Y. Mori, T. Sasaki, and T. Kobayashi, Jpn. J. Appl. Phys. 42, L1255 (2003).

8. C. Chen, Laser Focus World 40, 91 (2004).

9. Z. G. Hu, T. Higashiyama, M. Yoshimura, Y. Mori, and T. Sasaki, Z. Kristallogr.-New Cryst. Struct. 214, 433 (1999).
10. Z.-G. Hu, N. Ushiyama, Y. K. Yap, M. Yoshimura, Y. Mori, and T. Sasaki, J. Cryst. Growth 237-239, 654 (2002).

11. Z.-G. Hu, M. Yoshimura, Y. Mori, T. Sasaki, and K. Kato, Opt. Mater. 23, 353 (2003).

12. X. B. Hu, J. Y. Wang, C. Q. Zhang, X. G. Xu, C.-K. Loong, and M. Grimsditch, Appl. Phys. Lett. 85, 2241 (2004).

13. Z.-G. Hu, N. Ushiyama, Y. K. Yap, M. Yoshimura, Y. Mori, and T. Sasaki, Jpn. J. Appl. Phys. 40, L393 (2001).

14. L. Liu, C. Liu, X. Wang, Z. G. Hu, R. K. Li, and C. T. Chen, Solid State Sci. 11, 841 (2009).

15. Z. S. Lin, L. F. Xu, L. Liu, J. Xu, M. H. Lee, Z. Fang, and C. T. Chen, Phys. Rev. B: Condens. Matter 82, 035124 (2010).

16. G. Zimmerer, Nucl. Instrum. Methods Phys. Res., Sect. A. 308, 178 (1991).

17. Z. Lin, Z. Wang, C. Chen, S. K. Chen, and M.-H. Lee, J. Appl. Phys. 93, 9717 (2003).

18. I. N. Ogorodnikov, V. A. Pustovarov, A. V. Kruzhalov, L. I. Isaenko, M. Kirm, and G. Zimmerer, Phys. Solid State 42 (3), 464 (2000).

19. A. Yu. Kuznetsov, L. I. Isaenko, A. V. Kruzhalov, I. N. Ogorodnikov, and A. B. Sobolev, Phys. Solid State 41 (1), 48 (1999).

20. I. N. Ogorodnikov, V. A. Pustovarov, M. Kirm, A. V. Kruzhalov, and L. I. Isaenko, Phys. Solid State $\mathbf{4 3}$ (8), 1454 (2001).

21. V. Kisand, R. Kink, M. Kink, J. Maksimov, M. Kirm, and I. Martinson, Phys. Scr. 54, 542 (1996).

22. M. Kirm, G. Zimmerer, E. Feldbach, A. Lushchik, C. Lushchik, and F. Savikhin, Phys. Rev. B: Condens. Matter 60, 502 (1999).

23. I. N. Ogorodnikov, V. A. Pustovarov, and M. Kirm, Phys. Solid State 46 (5), 842 (2004).

24. V. T. Kuanyshev, T. A. Belykh, I. N. Ogorodnikov, B. V. Shulgin, M. K. Satybaldieva, and M. M. Kidibaev, Radiat. Meas. 33, 503 (2001).

25. I. N. Ogorodnikov, V. A. Pustovarov, A. V. Kruzhalov, L. I. Isaenko, M. Kirm, and G. Zimmerer, Phys. Solid State 42 (10), 1846 (2000).

26. I. N. Ogorodnikov, V. A. Pustovarov, M. Kirm, A. V. Kruzhalov, and L. I. Isaenko, Opt. Spectrosc. 92 (5), 702 (2002).

27. A. K. S. Song and R. T. Williams, Self-Trapped Excitons (Springer, Berlin, 1996).

28. I. N. Ogorodnikov, V. Yu. Ivanov, and A. V. Kruzhalov, Phys. Solid State 36 (11), 1748 (1994).

29. V. A. Pustovarov, V. Yu. Ivanov, M. Kirm, A. V. Kruzhalov, A. V. Korotaev, and G. Zimmerer, Phys. Solid State 43 (7), 1233 (2001).

30. B. R. Namozov, M. E. Fominich, R. I. Zakharchenya, and V. V. Myurk, Phys. Solid State 40 (5), 837 (1998).

31. W. B. White, M. Matsumura, D. G. Linnehan, T. Furukawa, and B. K. Chandrasekhar, Am. Mineral. 71, 1415 (1986).

Translated by A. Kazantsev 